\title{
GENERATORS FOR FINITE SIMPLE MOUFANG LOOPS
}

\author{
PETR VOJTĚCHOVSKÝ
}

\begin{abstract}
Moufang loops are one of the best-known generalizations of groups. There is only one countable family of nonassociative finite simple Moufang loops, arising from the split octonion algebras. We prove that every member of this family is generated by three elements, using the classical results on generators of unimodular groups.
\end{abstract}

Keywords: finite simple Moufang loops, Paige loops, minimal generators, generators for unimodular groups, projective unimodular groups.

\section{Finite Simple Moufang Loops}

A Moufang loop is a loop satisfying the identity $x(y(x z))=((x y) x) z$. Every element $x$ of a Moufang loop has a (unique) two-sided inverse $x^{-1}$; every two elements generate a group. Loops with the latter property are called diassociative [10]. The best-known Moufang loop is the multiplicative loop of nonzero elements in the standard 8-dimensional real octonion algebra $\mathbb{O}$. Surely the best-known finite Moufang loop is the 240-element loop $L$ of integral octonions of norm one [4].

In 1956, L. Paige [9] found one nonassociative finite simple Moufang loop for every finite field. Following E. Bannai and S. Song [3], we denote this Paige loop constructed over $F=G F(q)$ by $M^{*}(q)$. Let us give the most brief description of $M^{*}(q)$ now.

Consider the Zorn multiplication

$$
\left(\begin{array}{ll}
a & \alpha \\
\beta & b
\end{array}\right)\left(\begin{array}{ll}
c & \gamma \\
\delta & d
\end{array}\right)=\left(\begin{array}{cc}
a c+\alpha \cdot \delta & a \gamma+\alpha d-\beta \times \delta \\
\beta c+b \delta+\alpha \times \gamma & \beta \cdot \gamma+b d
\end{array}\right),
$$

where $a, b, c, d \in F, \alpha, \beta, \gamma, \delta \in F^{3}$, and where $\alpha \cdot \delta$ (resp. $\alpha \times \delta$ ) denotes the dot product (resp. cross product) of $\alpha$ and $\delta$. This is the same formula M. Zorn used to construct the split octonion algebra over $F$. The loop $M^{*}(q)$ consists of all matrices

$$
M=\left(\begin{array}{ll}
a & \alpha \\
\beta & b
\end{array}\right)
$$

with $\operatorname{det} M=a b-\alpha \beta=1$ that are multiplied according to (1), and where $M$ and $-M$ are identified. The neutral element of $M^{*}(q)$ is the identity matrix $I$, and the inverse of $M$ is

$$
M^{-1}=\left(\begin{array}{cc}
b & -\alpha \\
-\beta & a
\end{array}\right) .
$$

MSC: PRIMARY 20N05, SECONDARY 20F05. 
In 1987, M. Liebeck [8] proved that there are no other nonassociative finite simple Moufang loops. The loop $M^{*}(2)$ is exceptional in the sense that it shows up in the real algebra $\mathbb{O}$, too. Namely, $M^{*}(2)$ is isomorphic to the quotient of $L$ by its center $Z(L)=\{1,-1\}$ (see [4] once again).

Associative finite simple Moufang loops are finite simple groups. It is a remarkable fact that every finite simple group is 2-generated [2]; even more so, since no proof using only the simplicity is known. Instead, every family of finite simple groups must be investigated separately. Because of diassociativity, the nonassociative Paige loops cannot be 2-generated. It is reasonable to expect that a small number of generators will do. Indeed, it this paper we prove that:

Theorem 1.1. Every nonassociative finite simple Moufang loop is 3-generated.

Note that Theorem 1.1 was proved in [11] for all Paige loops $M^{*}(p), p$ a prime. Thus the main task of this paper is to cover the general case. We also present a simple proof for the prime case, and offer at least two generating sets for every $M^{*}(q)$. The reader who wants to establish 1.1 as quickly as possible should focus on 2.2, 2.3, and Section 3.

\section{Generators FOR $L_{2}(q)$}

The crucial observation concerning Paige loops is that $M^{*}(q)$ contains several copies of $L_{2}(q)=P S L_{2}(q)$. Given the canonical basis $e_{1}=(1,0,0), e_{2}=(0,1,0)$, $e_{3}=(0,0,1)$ of $F^{3}$, let $\phi_{i}: L_{2}(q) \longrightarrow M^{*}(q)$ be defined by

$$
\phi_{i}\left(\begin{array}{ll}
a & b \\
c & d
\end{array}\right)=\left(\begin{array}{cc}
a & b e_{i} \\
c e_{i} & d
\end{array}\right),
$$

and let $G_{i}$ be the image of $L_{2}(q)$ under $\phi_{i}$. Since the multiplication in $G_{i}$ coincides with the usual matrix multiplication (all cross products involved in (1) vanish), $\phi_{i}$ is an isomorphism $L_{2}(q) \longrightarrow G_{i}$.

This brings our attention to the classical results concerning generators for $L_{2}(q)$ and $S L_{2}(q)$. First of all, we have the Dickson Theorem:

Theorem 2.1 (L. E. Dickson, 1900). If $q \neq 9$ is an odd prime power or $q=2$, then $S L_{2}(q)$ is generated by

$$
\left(\begin{array}{ll}
1 & 1 \\
0 & 1
\end{array}\right),\left(\begin{array}{ll}
1 & 0 \\
\lambda & 1
\end{array}\right)
$$

where $\lambda$ is a primitive element of $G F(q)$.

The proof can be found in [6], and more recently in [7, pp. 44-55]. The statement of the theorem usually does not mention $q=2$, although it is apparently true for $q=2$, since $S L_{2}(2) \cong S_{3}$ is generated by any two involutions, in particular by (2).

A. A. Albert and J. Thompson proved [1, Lemma 8] that for any primitive element $\lambda$ of $G F(q), q>2$, the group $S L_{2}(q)$ is generated by $B,-B$, and $C$, 
where

$$
B=\left(\begin{array}{cc}
\lambda & 0 \\
0 & \lambda^{-1}
\end{array}\right), \quad C=\left(\begin{array}{cc}
0 & 1 \\
-1 & \lambda
\end{array}\right)
$$

We therefore have:

Proposition 2.2 (A. A. Albert, J. Thompson, 1959). Let $q>2$ be a prime power. Then $L_{2}(q)$ is generated by (3), where $\lambda$ is a primitive element of $G F(q)$.

The generators (3) are especially convenient for our purposes, because $\phi_{i}(B)=$ $B$ for every $i, 1 \leq i \leq 3$; but let us not get ahead of ourselves. It is practical to know some generators that do not involve a primitive element. For that matter, Coxeter and Moser argue in [5] that

Lemma 2.3. For every prime $p$, the group $L_{2}(p)$ is generated by

$$
\left(\begin{array}{ll}
1 & 0 \\
1 & 1
\end{array}\right), \quad\left(\begin{array}{cc}
0 & 1 \\
-1 & 0
\end{array}\right)
$$

\section{Generators For $M^{*}(q)$}

Our first result concerning $M^{*}(q)$ has nothing to do with the generators for $L_{2}(q)$. In its proof, we take advantage of the following lemma due to Paige:

Lemma 3.1 (L. Paige, 1956). $M^{*}(q)$ is generated by

$$
M_{\beta}=\left(\begin{array}{ll}
1 & 0 \\
\beta & 1
\end{array}\right), \quad M_{\beta}^{\prime}=\left(\begin{array}{cc}
1 & \beta \\
0 & 1
\end{array}\right),
$$

where $\beta$ runs over all nonzero vectors in $F^{3}$.

Proof. Combine Lemmas 4.2 and 4.3 of [9].

Proposition 3.2. $M^{*}(q)$ is generated by $G_{1} \cup G_{2} \cup G_{3}$.

Proof. Let $Q$ be the subloop of $M^{*}(q)$ generated by $G_{1} \cup G_{2} \cup G_{3}$. Thanks to Lemma [3.1, it suffices to prove that $Q$ contains all elements $M_{\beta}, M_{\beta}^{\prime}$, defined in (51). We show simultaneously that $M_{\beta} \in Q$ and $M_{\beta}^{\prime} \in Q$.

Let $k$ denote the number of nonzero entries of $\beta$. There is nothing to prove when $k \leq 1$. Suppose that $k=2$. Without loss of generality, let $\beta=(a, b, 0)$ for some $a, b \in F^{*}=F \backslash\{0\}$. Verify that

$$
\left(\begin{array}{cc}
1 & a e_{1} \\
0 & 1
\end{array}\right)\left(\begin{array}{cc}
1 & b e_{2} \\
0 & 1
\end{array}\right) \cdot\left(\begin{array}{cc}
1 & 0 \\
-a b e_{3} & 1
\end{array}\right)=\left(\begin{array}{cc}
1 & (a, b, 0) \\
0 & 1
\end{array}\right),
$$

and thus that $M_{\beta} \in Q$. Similarly, $M_{\beta}^{\prime} \in Q$. We can therefore assume that $Q$ contains all elements $M_{\beta}, M_{\beta}^{\prime}$ with $k \leq 2$.

Let $k=3, \beta=(a, b, c)$ for some $a, b, c \in F^{*}$. As

$$
\left(\begin{array}{cc}
1 & (a, b, 0) \\
0 & 1
\end{array}\right)\left(\begin{array}{cc}
1 & (0,0, c) \\
0 & 1
\end{array}\right) \cdot\left(\begin{array}{cc}
1 & 0 \\
(-b c, a c, 0) & 1
\end{array}\right)=\left(\begin{array}{cc}
1 & (a, b, c) \\
0 & 1
\end{array}\right)
$$

$M_{\beta}$ belongs to $Q$. Symmetrically, $M_{\beta}^{\prime} \in Q$, and we are done. 
In fact, $G_{1} \cup G_{2}$ already generates $M^{*}(q)$. The role of the cross product is especially apparent in the next Proposition.

Proposition 3.3. The subgroup $G_{3}$ is contained in the subloop of $M^{*}(q)$ generated by $G_{1} \cup G_{2}$. In particular, $M^{*}(q)$ is generated by $G_{1} \cup G_{2}$.

Proof. As it turns out, all we need are these two equations:

$$
\begin{aligned}
\left(\begin{array}{cc}
1 & 0 \\
\lambda e_{3} & 1
\end{array}\right) & =-\left(\begin{array}{cc}
0 & e_{2} \\
-e_{2} & 0
\end{array}\right)\left(\begin{array}{cc}
1 & \lambda e_{1} \\
-\lambda^{-1} e_{1} & 0
\end{array}\right) \cdot\left(\begin{array}{cc}
1 & e_{2} \\
-e_{2} & 0
\end{array}\right)\left(\begin{array}{cc}
1 & \lambda e_{1} \\
-\lambda^{-1} e_{1} & 0
\end{array}\right) \\
\left(\begin{array}{cc}
0 & e_{3} \\
-e_{3} & 0
\end{array}\right) & =\left(\begin{array}{cc}
0 & e_{1} \\
-e_{1} & 0
\end{array}\right)\left(\begin{array}{cc}
0 & -e_{2} \\
e_{2} & 0
\end{array}\right) .
\end{aligned}
$$

Note that the left hand sides of these equations are elements of $G_{3}$, whereas the right hand sides are products of elements of $G_{1} \cup G_{2}$. When $q=2$, we are done by Lemma 2.3. When $q>2$, observe that

$$
\left(\begin{array}{ll}
1 & 0 \\
\lambda & 1
\end{array}\right)\left(\begin{array}{cc}
0 & 1 \\
-1 & 0
\end{array}\right)=\left(\begin{array}{cc}
0 & 1 \\
-1 & \lambda
\end{array}\right)=C
$$

Since $B=\phi_{i}(B)$ for every $i, 1 \leq i \leq 3$, we are done by Proposition 2.2 .

Theorem 1.1 is now proved. When $q>2, M^{*}(q)$ is generated by $\phi_{1}(C), \phi_{2}(C)$ and $B=\phi_{1}(B)=\phi_{2}(B)$, by Propositions [2.2 and 3.3. When $q=2$, we are done by the main result of [11], Theorem 2.1 [11].

For the sake of completeness, allow us to present an alternative, simpler proof of [11, Theorem 2.1].

Proposition 3.4. [11, Theorem 2.1] Let $p$ be a prime. Then $M^{*}(p)$ is generated by

$$
U_{1}=\left(\begin{array}{cc}
1 & e_{1} \\
0 & 1
\end{array}\right), \quad U_{2}=\left(\begin{array}{cc}
1 & e_{2} \\
0 & 1
\end{array}\right), \quad X=\left(\begin{array}{cc}
0 & e_{3} \\
-e_{3} & 1
\end{array}\right)
$$

Proof. First check that

$$
\left(\begin{array}{ll}
1 & 0 \\
1 & 1
\end{array}\right)=\left(\begin{array}{cc}
0 & 1 \\
-1 & 0
\end{array}\right)\left(\begin{array}{ll}
1 & 1 \\
0 & 1
\end{array}\right)^{-1}\left(\begin{array}{cc}
0 & 1 \\
-1 & 0
\end{array}\right)^{-1} .
$$

Combine (44) and (6) to see that $L_{2}(p)$ is generated by

$$
U=\left(\begin{array}{ll}
1 & 1 \\
0 & 1
\end{array}\right), \quad V=\left(\begin{array}{cc}
0 & 1 \\
-1 & 0
\end{array}\right)
$$

Consequently, $M^{*}(p)$ is generated by $U_{1}=\phi_{1}(U), U_{2}=\phi_{2}(U), V_{1}=\phi_{1}(V)$, and $V_{2}=\phi_{2}(V)$. Now

$$
\begin{aligned}
& V_{2}=-\left(X U_{1} \cdot X U_{2}\right) \cdot X^{-1} U_{1}, \\
& V_{1}=-U_{1} U_{2} \cdot\left(V_{2} \cdot U_{1} X\right),
\end{aligned}
$$

and we are through. 


\section{More Generating Sets}

We would like to show how to obtain additional generating sets for $M^{*}(q)$. We take advantage of Proposition 3.3. Dickson's Theorem, and of the fact that $S L_{2}\left(2^{r}\right.$ ) (for $r>1$ ) is generated by

$$
D_{1}=\left(\begin{array}{ll}
1 & 1 \\
1 & 0
\end{array}\right), \quad D_{2}=\left(\begin{array}{cc}
\lambda & 0 \\
0 & \lambda^{-1}
\end{array}\right),
$$

where $\lambda$ is a primitive element of $G F\left(2^{r}\right)$. We leave the verification of (7) to the reader.

Since $\phi_{i}\left(D_{2}\right)=D_{2}$ for $i=1,2,3$, we immediately see from Proposition 3.3 that $M^{*}\left(2^{r}\right)$ (for $r>1$ ) is generated by $\phi_{1}\left(D_{1}\right), \phi_{2}\left(D_{1}\right)$ and $D_{2}$.

Proposition 4.1. Let $q \neq 9$ be an odd prime power or $q=2$. Then $M^{*}(q)$ is generated by

$$
\left(\begin{array}{cc}
1 & e_{1} \\
0 & 1
\end{array}\right),\left(\begin{array}{cc}
1 & e_{2} \\
0 & 1
\end{array}\right),\left(\begin{array}{cc}
0 & \lambda e_{3} \\
-\lambda^{-1} e_{3} & 1
\end{array}\right)
$$

where $\lambda$ is a primitive element of $G F(q)$.

Proof. Keeping Proposition 3.3 and Dickson's Theorem in mind, we only need to obtain the elements

$$
\left(\begin{array}{cc}
1 & 0 \\
\lambda e_{i} & 1
\end{array}\right)
$$

for $i=1,2$. Straightforward computation reveals that

$$
\begin{aligned}
\left(\begin{array}{cc}
1 & 0 \\
\lambda e_{1} & 1
\end{array}\right) & =-\left(\begin{array}{cc}
0 & \lambda e_{3} \\
-\lambda^{-1} e_{3} & 1
\end{array}\right)^{2}\left(\begin{array}{cc}
1 & e_{2} \\
0 & 1
\end{array}\right)\left(\begin{array}{cc}
0 & \lambda e_{3} \\
-\lambda^{-1} e_{3} & 1
\end{array}\right), \\
\left(\begin{array}{cc}
1 & 0 \\
\lambda e_{2} & 1
\end{array}\right)^{-1} & =-\left(\begin{array}{cc}
0 & \lambda e_{3} \\
-\lambda^{-1} e_{3} & 1
\end{array}\right)^{2}\left(\begin{array}{cc}
1 & e_{1} \\
0 & 1
\end{array}\right)\left(\begin{array}{cc}
0 & \lambda e_{3} \\
-\lambda^{-1} e_{3} & 1
\end{array}\right) .
\end{aligned}
$$

Note that the expressions on the right hand side can be evaluated in any order.

\section{REFERENCES}

[1] A. A. Albert, J. Thompson, Two-element generation of the projective unimodular group, Illinois J. Math. 3 (1959), 421-439.

[2] M. Aschbacher, R. Guralnick, Some applications of the first cohomology group, J. Algebra 90 (1984), no. 2, 446-460.

[3] E. Bannai, S. Song, The character tables of Paige's simple Moufang loops and their relationship to the character tables of $\operatorname{PSL}(2, q)$, Proc. London Math. Soc. (3) 58 (1989), no. 2, 209-236.

[4] H. S. M. Coxeter, Integral Cayley Numbers, Duke Mathematical Journal, Vol. 13, No. 4, December, 1946. Reprinted in H. S. M. Coxeter, Twelve Geometric Essays, Southern Illinois University Press, 1968. 
[5] H. S. M. Coxeter, W. O. J. Moser, Generators and relations for discrete groups. Fourth edition. Ergebnisse der Mathematik und ihrer Grenzgebiete [Results in Mathematics and Related Areas], 14. Springer-Verlag, Berlin-New York (1980).

[6] L. E. Dickson, Linear Groups with an Exposition of the Galois Field Theory, Teubner (1901); reprinted by Dover (1958).

[7] D. Gorenstein, Finite groups. Second edition. Chelsea Publishing Co., New York (1980).

[8] M. W. Liebeck, The classification of finite simple Moufang loops. Math. Proc. Cambridge Philos. Soc. 102 (1987), no. 1, 33-47.

[9] L. Paige, A Class of Simple Moufang Loops, Proceedings of the American Mathematical Society, Vol. 7, Issue 3, 471-482, June 1956.

[10] H. O. Pflugfelder, Quasigroups and Loops: Introduction, (Sigma series in pure mathematics; 7), Heldermann Verlag Berlin (1990).

[11] P. Vojtěchovský, Generators of Nonassociative Simple Moufang Loops over Finite Prime Fields, J. of Algebra 241 (2001), 186-192.

Department of Mathematics, Iowa State University, Ames, IA, U.S.A.

E-mail address: petr@iastate.edu 